\title{
Ensamble de murciélagos en el valle de Ite, región Tacna, Perú
}

\author{
Assembly of bats in the valley of Ite, region Tacna, Peru \\ Teresa Lanchipa Ale1, Giovanni Aragón Alvarado ${ }^{*}$
}

\section{RESUMEN}

El valle de Ite en la región Tacna, al sur del Perú, es el lugar con mayor cantidad de especies de murciélagos reportados hasta ahora. Todos ellos conforman un gremio de insectívoros que explotan el mismo sustrato trófico. El propósito planteado fue determinar las características del ensamble trófico en este valle. Se establecieron 8 estaciones utilizando redes de neblina, que se desplegaron por 12 horas, por 3 días por mes, durante ocho meses. Se analizó contenido de fecas y contenido estomacal; y mediante trampas luz se capturó la fauna entomológica nocturna del valle. Se colectaron cuatro especies de murciélagos: Histiotus montanus con 9 categorías alimenticias, Myotis atacamensis con 15, Mormopterus kalinowskii con 16 y para Tadarida brasieliensis sólo se reportaron lepidópteros y coleópteros. Presentaron un nicho trófico estrecho y bajo porcentaje de similitud. La relación de sus dietas con la oferta de la fauna entomológica del valle determina una alta búsqueda de los recursos específicos.

Palabras clave: dieta, gremio, ensamble, categorías tróficas, nicho trófico.

\begin{abstract}
The valley of Ite in the Tacna region, in southern Peru, is the place with the highest number of bat species reported so far. All of them make up a guild of insectivores exploiting the same trophic substrate. The proposed purpose was to determine the characteristics of the trophic assemblage in this valley. Eight stations were established using fog nets, which were deployed for 12 hours, for 3 days per month, for eight months, content of feces and stomach was analyzed; by means of light traps, the nocturnal entomological fauna of the valley was captured. Four species of bats were collected: Histiotus montanus with 9 food categories, Myotis atacamensis with 15, Mormopterus kalinowskii with 16 and for Tadarida brasieliensis only lepidoptera and coleoptera were reported. They presented a narrow trophic niche and low percentage of similarity. The relationship of their diets with the offer of entomological fauna of the valley determines a high search for specific resources.
\end{abstract}

Key words: diet, guild, ensemble, trophic categories, trophic niche.

\section{Introducción}

Los murciélagos presentan variedad de fuentes de alimentación como frutos, néctar, sangre, insectos, etc., siendo la mayoría insectívoros, razón por la cual son considerados como controladores naturales ya que en su dieta se hallan plagas agrícolas (Swier, 2003). Se ha determinado que la composición, variación temporal, proporción y la frecuencia de las presas permiten reconocer que seleccionan sus presas (Hodgkison et al., 2004; Lee \& Lee, 2005), y que la selección se da con el sexo, edad, estado reproductivo y entre poblaciones (Moreno \& Roa, 2005). La evaluación de la amplitud del nicho trófico permite reconocer el traslape de dietas en las preferencias alimentarias (Lou \& Yurrita, 2005; Debelica et al., 2006; Loayza, 2006; López \& Vaughan, 2007). Por otro lado, al conocer los hábitos alimenticios de los murciélagos se puede analizar su comportamiento forrajero (Burles et al., 2008). Para este trabajo se considera como ensamble al conjunto de murciélagos que usan el mismo recurso alimenticio: los murciélagos insectívoros del valle de Ite. El ensamble en su composición y estructura es el resultado de la interacción entre las especies de murciélagos, el ambiente y el proceso histórico que lo origina. Existe una diversidad de ensamblajes de murciélagos y variedad en sus interacciones y que no se manifiestan de la misma manera en los

\footnotetext{
1 Universidad Nacional Jorge Basadre Grohmann. Tacna, Perú.

* Autor por correspondencia: garagona@unjbg.edu.pe
} 
diferentes ecosistemas (Estrada-Villegas et al., 2010). En nuestra zona desértica debe ser baja la diversidad de ensamblajes.

En la región de Tacna se tiene registro de nueve especies de murciélagos (Aragón y Aguirre, 2014), ocho insectívoras y una sola entomófaga. En el valle de Ite se han reportado cuatro especies: Histiotus montanus, Myotis atacamensis, Mormopterus kalinowskii y Tadarida brasiliensis. Todos ellos conforman un gremio de insectívoros que explotan la misma fuente alimenticia. El propósito del presente trabajo fue determinar las características tróficas en la comunidad de murciélagos en el valle de Ite, así como la amplitud de nicho y su superposición entre las distintas especies.

\section{Materiales y métodos}

Las actividades agropecuarias caracterizan al valle de Ite, ubicado en la provincia Jorge Basadre en la región Tacna, Perú (175 m de altitud, 70 57'47" O y $17^{\circ} 50$ '27" S). Se establecieron 6 estaciones de muestreo, y en cada una se instalaron 4 redes de 12 × 2,5 m, a una altura de $0,5 \mathrm{~m}$ del suelo. Se desplegaron desde las 17:00 hasta las 5:00 horas del día siguiente. La frecuencia fue 3 días/mes y 12 horas/día, durante ocho meses. La ubicación taxonómica se realizó por la caracterización morfométrica mediante las descripciones de Albuja, 1999; Zeballos \& López, 2002; Gardner, 2008; y Aguirre et al. 2009. Luego fueron colocados en bolsas de tela de 2 a 4 horas hasta obtener muestras fecales y posteriormente liberarlos. Las fecas se llevaron al laboratorio para los análisis correspondientes. En el área de estudio mediante trampas luz se colectaron insectos como referencia, para determinar la disponibilidad del recurso. Las trampas luz se ubicaron aproximadamente a $40 \mathrm{~m}$ de las redes evitando interferencia lumínica. Los insectos capturados se conservaron en etanol al $70 \%$ y fueron transportados a laboratorio para su caracterización. Estos insectos se usaron como referencia para reconocer los restos obtenidos en las fecas, y para ello cada individuo fue fraccionado (tarsos, alas, antenas, etc.). Posteriormente se ablandaron mediante una solución de hidrato de cloral con ácido fénico en relación 1/1, por 24 horas. Luego se les montó en portaobjetos en bálsamo de Canadá y cubreobjetos, y finalmente fueron etiquetadas. Semejante tratamiento se realizó a los restos de insectos presentes en las fecas.
Las muestras fecales se trataron con etanol al $70 \%$ por 24 horas y luego se analizaron al estereoscopio (16X). Todos los fragmentos identificables fueron separados y determinados por comparación y mediante claves entomológicas de Mc Gavin (2000) y Gillot (2005). Semejante tratamiento se realizó con el contenido estomacal del único ejemplar de T. brasiliensis.

Con base en el porcentaje de ocurrencia de las presas por fecha se determinó la estructura y composición de las dietas. La amplitud de nicho alimenticio se determinó mediante el Índice de Levins y Smith. El solapamiento de nicho se calculó mediante el índice de MacArthur y Levins, modificado por Pianka y el índice simplificado de Morisita (Krebs, 1999).

\section{Resultados y discusión}

Se capturaron 36 murciélagos pertenecientes a cuatro especies: Histiotus montanus (9), Myotis atacamensis (18), Mormopterus kalinowskii (8) y Tadarida brasiliensis (1) (Tabla 1).

Se colectaron 22.981 individuos de artrópodos pertenecientes a 42 familias de la clase Insecta y 2 familias de la clase Arachnida.

El orden Díptera fue representado con $43,45 \%$; Coleóptera el 29,33\%; Lepidóptera con el 15,64\%; Hemíptera con 5,52\%. Los demás órdenes estuvieron representados con menos del 2\%. El orden Acarina con el $1,09 \%$ y Araneae con el $0,17 \%$. (Tabla 2, Figura 1).

Se obtuvieron 163 fecas de Myotis atacamensis, 86 de Mormopterus kalinowskii, 76 de Histiotus montanus, ninguna feca en Tadarida brasiliensis, paraun total de 325 colectadas durante el período de estudio. De Myotis atacamensis se analizaron 163 fecas y se hallaron 15 categorías alimenticias. Resalta el orden Himenóptera (30\%) que tiene

Tabla 1. Murciélagos capturados en el valle de Ite.

\begin{tabular}{lcr}
\hline \multicolumn{1}{c}{ Especie } & $\begin{array}{c}\text { Cantidad de } \\
\text { Individuos } \\
\text { Capturados }\end{array}$ & Porcentaje \\
\hline Histiotus montanus & 9 & 25 \\
Myotis atacamensis & 18 & 51 \\
Mormopterus kalinowskii & 8 & 22 \\
Tadarida brasiliensis & 1 & 2 \\
Total & 36 & 100 \\
\hline
\end{tabular}


Tabla 2. Artrópodos capturados con trampas luz y su presencia en la dieta de los murciélagos.

\begin{tabular}{|c|c|c|c|c|}
\hline Clase & Orden & Familia & Especie sin Identificar S/I & Presente en la dieta de: \\
\hline \multirow{3}{*}{ Arachnida } & Acarina & Tarsonemidae & Polyphagotarsonemus latus & $\begin{array}{l}\text { M. atacamensis } \\
\text { M. kalinowskii }\end{array}$ \\
\hline & \multirow{2}{*}{ Araneae } & $\underline{\text { Salticidae }}$ & $\mathrm{S} / \mathrm{I}$ & \\
\hline & & $\mathrm{S} / \mathrm{I}$ & $\mathrm{S} / \mathrm{I}$ & \\
\hline \multirow{38}{*}{ Insecta } & \multirow{16}{*}{ Coleóptera } & Curculionidae & $\mathrm{S} / \mathrm{I}$ & \\
\hline & & Anthicidae & $\mathrm{S} / \mathrm{I}$ & \\
\hline & & Elateridae & $\mathrm{S} / \mathrm{I}$ & \\
\hline & & Scarabaeidae & Labarrus pseudolividus & $\begin{array}{l}\text { M. atacamensis } \\
\text { M. kalinowskii }\end{array}$ \\
\hline & & $\underline{\text { Scarabaeidae }}$ & Botinus maimon & \\
\hline & & $\underline{\text { Scarabaeidae }}$ & $\mathrm{S} / \mathrm{I}$ & \\
\hline & & Tenebrionidae & $\mathrm{S} / \mathrm{I}$ & $\begin{array}{l}\text { M. atacamensis } \\
\text { M. kalinowskii }\end{array}$ \\
\hline & & $\underline{\text { Nitidulidae }}$ & $\mathrm{S} / \mathrm{I}$ & M. kalinowskii \\
\hline & & Bostrichidae & Neoterius farmarei & \\
\hline & & Carabidae & Bradycellus sp. & \\
\hline & & $\underline{\text { Carabidae }}$ & $\mathrm{S} / \mathrm{I}$ & M. atacamensis \\
\hline & & Cicindellidae & Cicindella peruviana & Histiotus montanus \\
\hline & & Meloidae & $\mathrm{S} / \mathrm{I}$ & \\
\hline & & Anobiidae & $\mathrm{S} / \mathrm{I}$ & \\
\hline & & $\underline{\text { Staphylinidae }}$ & $\mathrm{S} / \mathrm{I}$ & \\
\hline & & Hydrophilidae & Enochrus sp. & \\
\hline & \multirow{5}{*}{ Lepidóptera } & Pyralidae & $\mathrm{S} / \mathrm{I}$ & $\begin{array}{l}\text { H. montanus } \\
\text { M. atacamensis }\end{array}$ \\
\hline & & Gelechiidae & $\mathrm{S} / \mathrm{I}$ & $\begin{array}{l}\text { H. montanus } \\
\text { M. atacamensis }\end{array}$ \\
\hline & & Noctuidae & $\mathrm{S} / \mathrm{I}$ & $\begin{array}{l}\text { H. montanus } \\
\text { M. atacamensis }\end{array}$ \\
\hline & & $\mathrm{S} / \mathrm{I}$ & $\mathrm{S} / \mathrm{I}$ & $\begin{array}{l}\text { H. montanus } \\
\text { M. atacamensis }\end{array}$ \\
\hline & & Braconidae & $\mathrm{S} / \mathrm{I}$ & \\
\hline & \multirow{4}{*}{ Hymenóptera } & Ichneumonidae & Enicospilus sp. & \\
\hline & & Formicidae & $\mathrm{S} / \mathrm{I}$ & \\
\hline & & Scoliidae & $\mathrm{S} / \mathrm{I}$ & \\
\hline & & $\mathrm{S} / \mathrm{I}$ & $\mathrm{S} / \mathrm{I}$ & M. atacamensis \\
\hline & \multirow{13}{*}{ Díptera } & Muscidae & $\mathrm{S} / \mathrm{I}$ & $\begin{array}{l}\text { H. montanus } \\
\text { M. atacamensis } \\
\text { M. kalinowskii }\end{array}$ \\
\hline & & Muscidae & Stomoxys calcitrans & \\
\hline & & Simulidae & $\mathrm{S} / \mathrm{I}$ & $\begin{array}{l}\text { M. kalinowskii } \\
\text { M. kalinowskii }\end{array}$ \\
\hline & & Chyronomidae & $\mathrm{S} / \mathrm{I}$ & \\
\hline & & Calliphoridae & $\mathrm{S} / \mathrm{I}$ & \\
\hline & & Culicidae & $\mathrm{S} / \mathrm{I}$ & M. kalinowskii \\
\hline & & Asilidae & Erax sp & \\
\hline & & Drosophilidae & $\mathrm{S} / \mathrm{I}$ & $\begin{array}{l}\text { M. atacamensis } \\
\text { M. kalinowskii }\end{array}$ \\
\hline & & Phoridae & $\mathrm{S} / \mathrm{I}$ & \\
\hline & & Otitidae & Euxesta sp. & \\
\hline & & Bombyliidae & $\mathrm{S} / \mathrm{I}$ & \\
\hline & & Tephritidae & Ceratitis capitata & \\
\hline & & $\mathrm{S} / \mathrm{I}$ & & $\begin{array}{l}\text { M. atacamensis } \\
\text { M. kalinowskii }\end{array}$ \\
\hline
\end{tabular}


Continuación Tabla 2

\begin{tabular}{|c|c|c|c|c|}
\hline Clase & Orden & Familia & Especie sin Identificar S/I & Presente en la dieta de: \\
\hline \multirow{15}{*}{ Insecta } & \multirow{12}{*}{ Hemíptera } & Miridae & Hyalochloria denticornis & \\
\hline & & Miridae & S/I & \\
\hline & & Nabidae & Nabis punctipennis & $\begin{array}{l}\text { M. atacamensis } \\
\text { M. kalinowskii }\end{array}$ \\
\hline & & Nabidae & $\mathrm{S} / \mathrm{I}$ & \\
\hline & & Coreidae & $\mathrm{S} / \mathrm{I}$ & $\begin{array}{l}\text { M. atacamensis } \\
\text { M. kalinowskii }\end{array}$ \\
\hline & & Lygaeidae & S/I & \\
\hline & & Neididae & Jalysus spinosus & \\
\hline & & Psilidae & $\mathrm{S} / \mathrm{I}$ & \\
\hline & & Anthocoridae & Orius sp & \\
\hline & & Delphacidae & Peregrinus maidis & \\
\hline & & Delphacidae & S/I & \\
\hline & & S/I & $\mathrm{S} / \mathrm{I}$ & \\
\hline & Homóptera & Cicadellidae & S/I & M. atacamensis \\
\hline & \multirow{2}{*}{ Neuróptera } & Chrysopidae & Chrysoperla sp. & \\
\hline & & Myrmeleontidae & S/I & \\
\hline
\end{tabular}

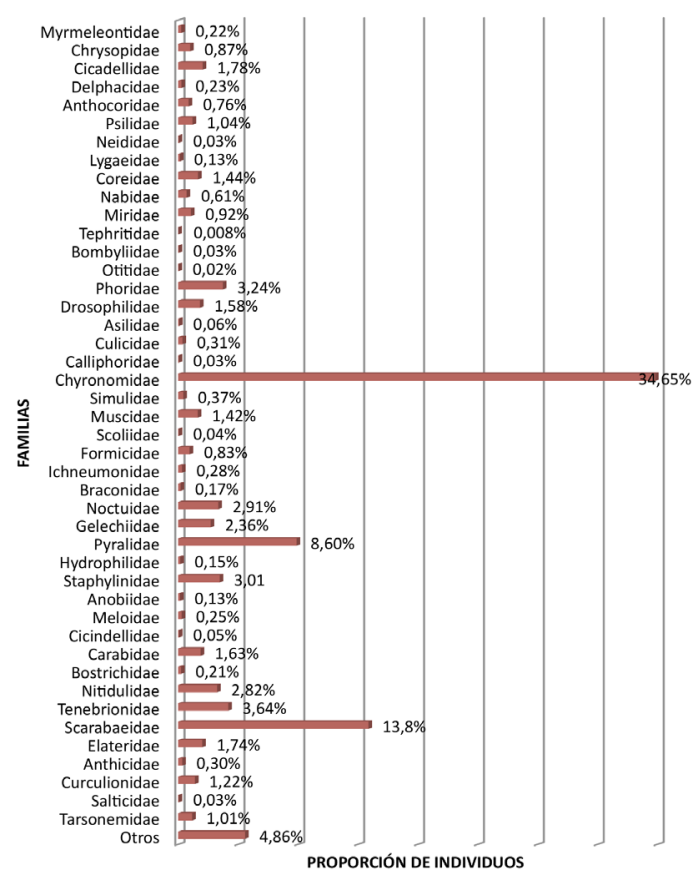

Figura 1. Porcentaje de familias de artrópodos capturados con trampas luz en el valle de Ite.

mayormente actividad diurna, Díptera con las familias Drosophilidae $(12,46 \%)$ y Muscidae (1.72\%), entre otros dípteros no identificados, todos estos de actividad diurna principalmente. El orden Lepidóptera con las familias Pyralidae $(9,15 \%)$, Noctuidae $(1,85 \%)$, Gelechiidae $(2,51 \%)$. Hemíptera con las familias Coreidae $(4,77 \%)$ y
Nabidae (0,13\%). Y en menor proporción los órdenes Coleóptera, Homóptera y Acarina. En este último orden se reportó el ácaro Polyphagotarsonemus latus $(14,07 \%)$, importante plaga agrícola de diversos cultivos como el ají. Ello resalta la función de controlador biológico de $M$. atacamensis con actividad crepuscular (Tabla 3).Se analizaron 86 fecas de Mormopterus kalinowskii determinándose 16 categorías alimenticias semejante al rango de M. atacamensis. Resalta el orden Díptera con las familias Chyronomidae (14,07\%), Drosophilidae $(17,36 \%)$, Simulidae $(4,49 \%)$, Muscidae $(3,89 \%)$, Culicidae (2,39\%), y sin identificar (14,37\%). El orden Coleóptera $(18,8 \%)$ con la especie Labarrus pseudolividus $(15,56 \%)$ de la familia Scarabaeidae, además de las familias Tenebrionidae $(2,39 \%)$ y Nitidulidae $(0,89 \%)$. El orden Hemíptera $(4,47 \%)$ con las familias Coreidae (2,69\%), Nabidae $(0,29 \%)$, y sin identificar (1,49\%). (Tabla 4).

En Histiotus montanus se registraron 6 categorías alimenticias en 76 fecas analizadas, siendo el orden Lepidóptera, el principal componente de su dieta $(91,3 \%)$, concordante con lo mencionado por Canals et al. (2005). En menor proporción los órdenes Heteróptera $(2,31 \%)$, Coleóptera $(1,73)$, Odonata $(0,57 \%)$ y Díptera $(1.15 \%)$, semejante a lo reportado por Aguirre (2007). Los organismos que forman parte de su dieta son de vuelo bajo y están presentes en las zonas de cultivo, lo que permite inferir que el forrajeo lo realiza a una media altura y en sitios abiertos. (Tabla 5). 
Tabla 3. Porcentaje de ocurrencias de los componentes alimenticios en las muestras fecales de Miotis atacamensis. (Frecuencia de Ocurrencia y Porcentaje de Ocurrencia).

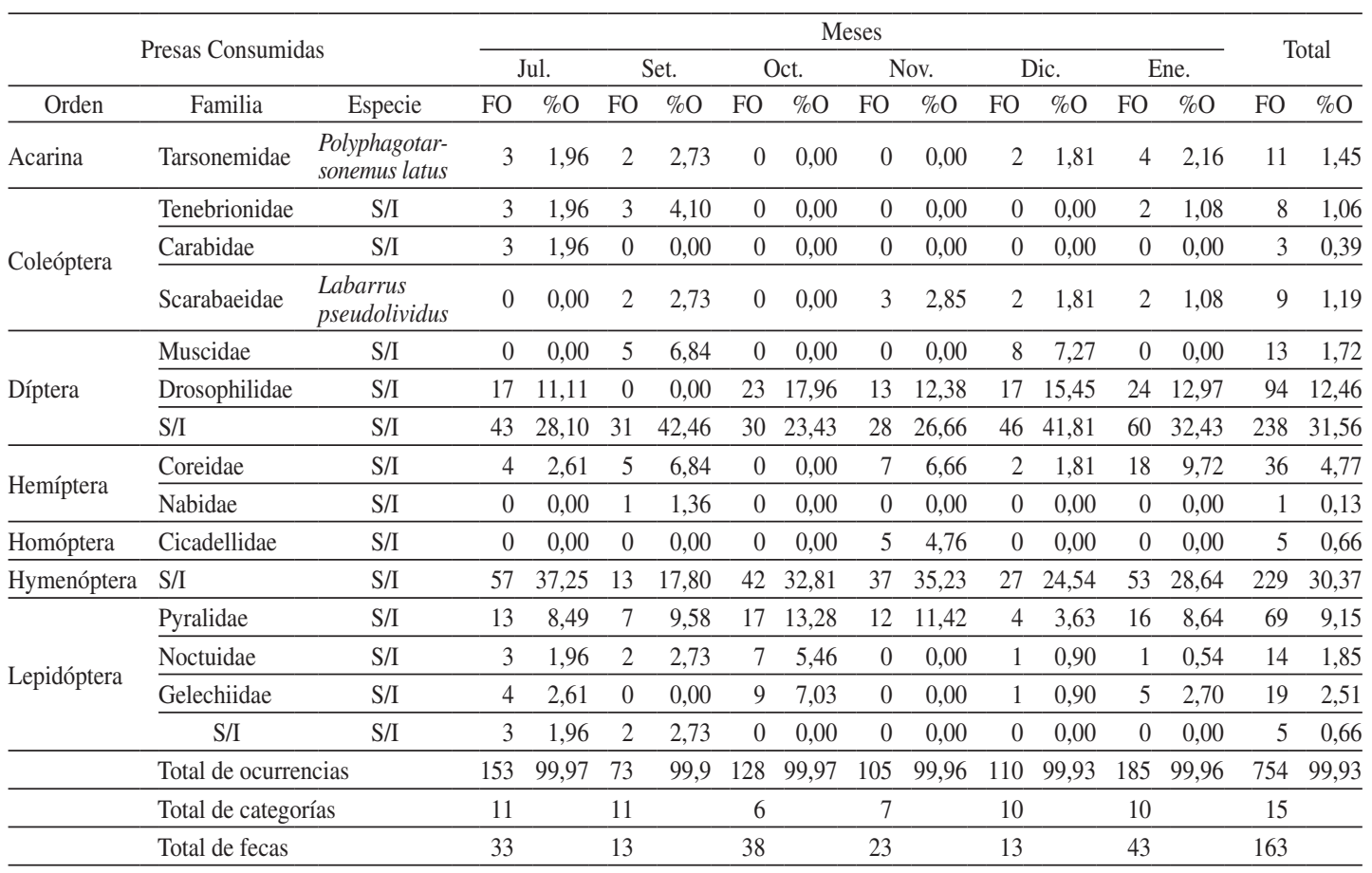

Tabla 4. Frecuencia y porcentaje de ocurrencias de los componentes alimenticios en las muestras fecales de Mormopterus kalinowskii.

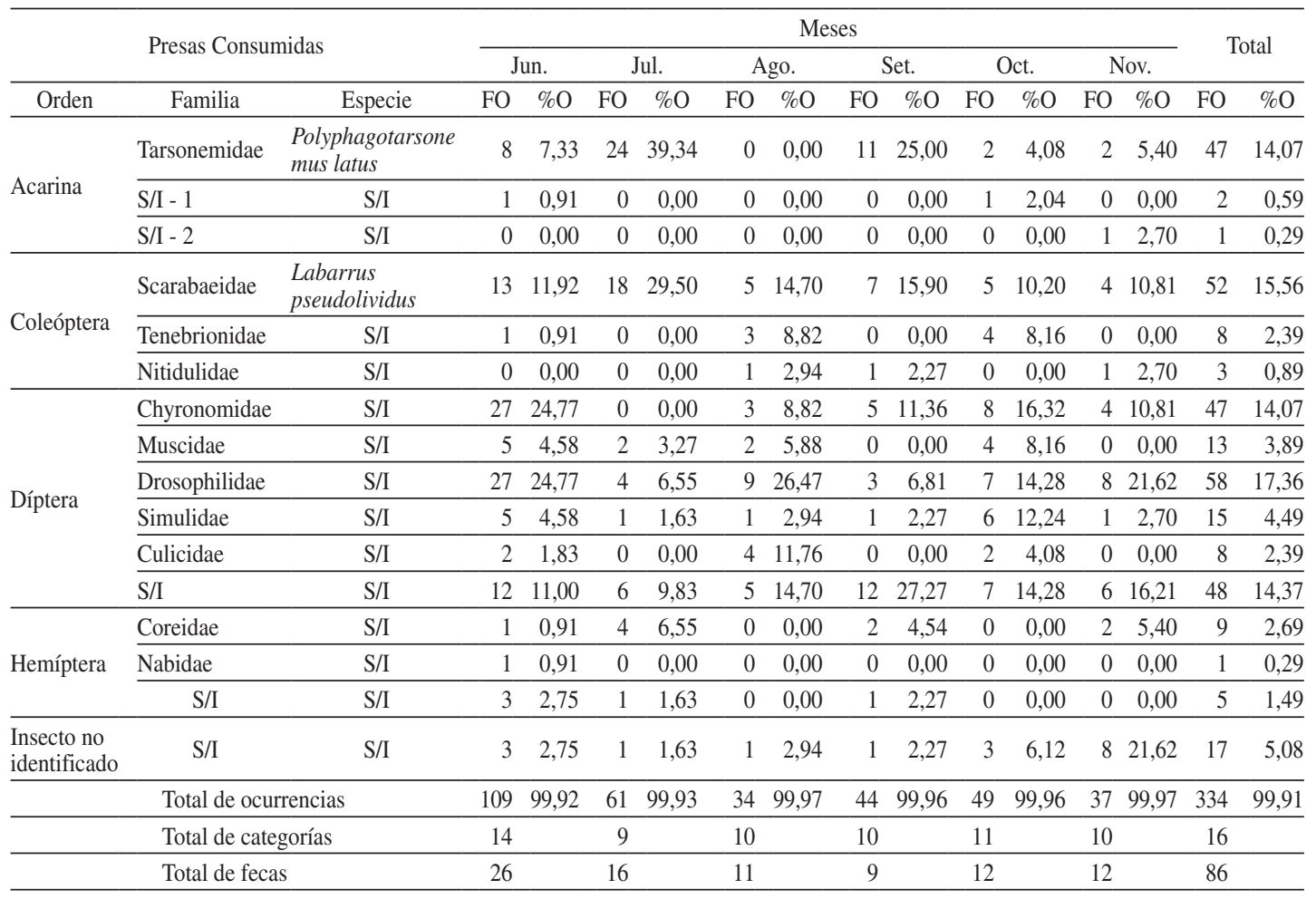


Tabla 5. Frecuencia y porcentaje de ocurrencias de los componentes alimenticios en las muestras fecales de Histiotus montanus.

\begin{tabular}{|c|c|c|c|c|c|c|c|c|c|c|c|c|c|c|}
\hline \multirow{2}{*}{\multicolumn{3}{|c|}{ Presas Consumidas }} & \multicolumn{10}{|c|}{ Meses } & \multirow{2}{*}{\multicolumn{2}{|c|}{ Total }} \\
\hline & & & \multicolumn{2}{|r|}{ Jul. } & \multicolumn{2}{|c|}{ Set. } & \multicolumn{2}{|c|}{ Oct. } & \multicolumn{2}{|c|}{ Nov. } & \multicolumn{2}{|c|}{ Ene. } & & \\
\hline Orden & Familia & Especie & FO & $\% 0$ & FO & $\% 0$ & FO & $\% \mathrm{O}$ & FO & $\% 0$ & FO & $\% 0$ & FO & $\% 0$ \\
\hline Coleóptera & Cicindellidae & Cicindella peruviana & 0 & 0 & 0 & 0 & 0 & 0 & 2 & 5,26 & 1 & 1,66 & 3 & 1,73 \\
\hline Díptera & Muscidae & $\mathrm{S} / \mathrm{I}$ & 0 & 0 & 0 & 0 & 0 & 0 & 0 & 0 & 2 & 3,33 & 2 & 1,15 \\
\hline \multirow{4}{*}{ Lepidóptera } & $\underline{\text { Pyralidae }}$ & S/I & 4 & 30,76 & 3 & 27,27 & 17 & 33,33 & 13 & 34,21 & 17 & 28,33 & 54 & 31,21 \\
\hline & Noctuidae & S/I & 1 & 7,69 & 1 & 9,09 & 7 & 13,72 & 5 & 13,15 & 9 & 15,00 & 23 & 13,29 \\
\hline & Gelechiidae & S/I & 2 & 15,38 & 1 & 9,09 & 5 & 9,80 & 0 & 0 & 0 & 0 & 8 & 4,62 \\
\hline & S/I & S/I & 5 & 38,46 & 6 & 54,54 & 20 & 39,21 & 16 & 42,10 & 26 & 43,33 & 73 & 42,19 \\
\hline Heteróptera & S/I & S/I & 1 & 7,69 & 0 & 0 & 1 & 1,96 & 0 & 0 & 2 & 3,33 & 4 & 2,31 \\
\hline Odonata & S/I & S/I & 0 & 0 & 0 & 0 & 1 & 1,96 & 0 & 0 & 0 & 0 & 1 & 0,57 \\
\hline Insecto no identificado & $\mathrm{S} / \mathrm{I}$ & S/I & 0 & 0 & 0 & 0 & 0 & 0 & 2 & 5,26 & 3 & 5,00 & 5 & 2,89 \\
\hline Total de ocurrencias & & & 13 & 99,98 & 11 & 99,99 & 51 & 99,98 & 38 & 99,98 & 60 & 99,98 & 173 & 99,96 \\
\hline Total de categorías & & & 5 & & 4 & & 6 & & 5 & & 7 & & 9 & \\
\hline Total de fecas & & & 7 & & 10 & & 17 & & 21 & & 38 & & 93 & \\
\hline
\end{tabular}

Se evidencia la actividad crepuscular de los quirópteros, como lo señala Sosa et al. (1996) al reportar en la dieta Chyronomidae y Drosophilidae, típicamente diurnas. La gran abundancia de Quironómidos representados por mosquitos hematófagos, potenciales reservorios naturales de V. cholerae (Halpern et al. 2003), así como de Simúlidos y Culícidos también hematófagos, en la dieta de M. kalinowskii y M. atacamensis, resalta su valor como controladores biológicos en este valle, especialmente por el reporte del ácaro plaga Polyphagotarsonemus latus en la dieta de M. atacamensis y M. kalinowskii que confirman el impacto positivo ecológico y económico que representan estas especies en este agroecosistema (Kunz, 2004).

Se capturó un solo ejemplar de Tadarida brasiliensis que no generó fecas. Sin embargo se sabe que es una especie generalista, y sus principales presas son coleópteros y lepidópteros, y en menor proporción dípteros, himenópteros, neurópteros, hemípteros, homópteras, odonatos y hormigas (McWilliams, 2005; Matthews, 2010). En el análisis del contenido estomacal se identificaron restos de lepidópteros y coleópteros, datos no tomados en cuenta para el análisis por tener poca representatividad.

La amplitud de nicho trófico de $H$. montanus (Levins $=0,3225 ;$ Smith $=0,8036)$, M. atacamensis
(Levins $=0,2552 ;$ Smith $=0,7628)$, M. kalinowskii (Levins $=0,4819$; Smith $=0,8492$ ) presenta valores relativamente bajos, lo que indicaría que son especies especialistas. (Tabla 6).

Los índices de similitud proporcional de las dietas en este ensamble (Tabla 8) testimonian bajo nivel de superposición de nicho (Tabla 7) entre las tres especies analizadas mediante los índices de Mac Arthur, Levins y Morisita. Entre H. montanus y $M$. Kalinowskii, así como entre H. montanus y $M$. atacamensis, presentan baja competencia por las presas. En cambio entre M. atacamensis y M. kalinowskii se percibe un solapamiento relativamente significativo por compartir varias categorías alimenticias (Tabla 8).

Es inobjetable que compartir hábitat condiciona el consumo de presas similares. Sin embargo la disimilitud puede estar condicionada por tamaño de la presa, estructura del exoesqueleto de la presa, forma y tamaño del ala y uropatagio (especialización morfológica), así como riqueza y/o abundancia de presas, estrategias de caza, tipo de hábitat, etc., variables que determinan la estructura de una comunidad biológica (Acosta, 2002).

La baja amplitud del nicho trófico, baja diversidad de murciélagos y la relativa alta variabilidad y abundancia del recurso alimenticio permiten expresar que es baja también la 
Tabla 6. Valor de amplitud de nicho trófico.

\begin{tabular}{cccc}
\hline \multicolumn{1}{c}{ Índice } & Histiotus montanus & Myotis atacamensis & Mormopterus kalinowskii \\
\hline Índice de Levins & 0,3225 & 0,2552 & 0,4819 \\
Índice de Smith & 0,8036 & 0,7628 & 0,8492 \\
\hline
\end{tabular}

Tabla 7. Valor de solapamiento de nicho trófico entre las especies de murciélagos.

\begin{tabular}{lccc}
\hline \multicolumn{1}{c}{ Pares de Especies } & Índice de MacArthur y Levins & Índice de Pianka & Índice Simplificado de Morisita \\
\hline H. montanus - M. atacamensis & $\begin{array}{l}M_{j k}=0,1183 \\
M_{k j}=0,1599\end{array}$ & 0,1375 & 0,1360 \\
\hline H. montanus - M. kalinowskii & $\begin{array}{l}M_{j k}=0,0064 \\
M_{k j}=0,0155\end{array}$ & 0,0100 & 0,0091 \\
\hline M. atacamensis - M. kalinowskii & $\begin{array}{l}M_{j k}=0,3324 \\
M_{k j}=0,5945\end{array}$ & 0,4445 & 0,4264 \\
\hline
\end{tabular}

Tabla 8. Índice de Similitud Proporcional entre las dietas de Histiotus montanus, Myotis atacamensis y Mormopterus kalinowskii.

\begin{tabular}{lcr}
\hline Pares de Especies & $\begin{array}{c}\text { Índice de Similitud } \\
\text { proporcional }\end{array}$ & $\%$ \\
\hline H. montanus - M. atacamensis & 0,1682 & 16,8 \\
H. montanus - M. kalinowskii & 0,0410 & 4,1 \\
M. atacamensis - M. kalinowskii & 0,3515 & 35,1 \\
\hline
\end{tabular}

superposición trófica en esta comunidad de murciélagos (Tabla 9). (Alvares et al., 1990).

\section{Conclusiones}

Se determinó que el gremio de murciélagos del valle de Ite está constituido por Histiotus, montanus con 9 categorías alimenticias, Myotis atacamensis con 15, Mormopterus kalinowskii con 16 y Tadarida brasieliensis con un solo ejemplar en el que se determinó presencia de lepidópteros y coleópteros. Que en el valle de Ite se aprecie una baja diversidad de murciélagos con bajo traslape de nicho trófico ante la amplia diversidad del sustrato alimenticio, puede explicarse en el sentido de que la teoría del ensamble menciona que su composición y estructura son producto de las condiciones
Tabla 9. Índice de Similitud Proporcional entre las dietas de los murciélagos y la oferta de insectos disponibles.

\begin{tabular}{llr}
\hline \multicolumn{1}{c}{ Especie } & Oferta Disponible & $\%$ \\
\hline H. montanus & 0,0869 & 8,69 \\
M. atacamensis & 0,2376 & 23,76 \\
M. kalinowskii & 0,3829 & 38,29 \\
\hline
\end{tabular}

ambientales y su proceso histórico: el nombre de este valle es el acrónimo de "Irrigación de Tierras Eriazas", cuyas actividades agrícolas son de reciente data. El valle nace luego de la reincorporación de Tacna al Perú en 1929. Después de la guerra fueron los explebiscitarios tacneños los primeros colonos de las áridas planicies de este valle, quienes no avanzaron en su propósito por las características edafo-climáticas de la zona. Posteriormente fue poblada por agricultores arequipeños (1960), que generaron las condiciones actuales. Por tanto, la historia de este valle es reciente, lo que debe ser la causa de la baja diversidad de murciélagos y la baja superposición de sus nichos tróficos. Estos resultados permiten interpretar que estas especies tienen características de especialistas y ello conduce a asumir que deben realizar desplazamientos y esfuerzos relevantes en la búsqueda del alimento. 


\section{Literatura Citada}

Acosta, M.

2002. Nicho y evolución. Facultad de Biología. Universidad de La Habana. Revista Biología, 16(1) 3-7.

Aguirre, L.F.; Vargas, A., Solari. S.

2009. Clave de campo para la identificación de los murciélagos de Bolivia. Centro de Estudios en Biología Teórica y Aplicada. Cochabamba, Bolivia, 38 p.

Albuja, L.

1999. Murciélagos del Ecuador. 2da. ed. Escuela Politécnica Nacional. Quito, 288 p.

Álvarez, B., F. Amezcua \& M. Álvarez-Rubio.

1990. Análisis de la diversidad, amplitud y traslape del nicho en la comunidad de peces del sistema Teacapan - Agua Brava, Nayarit, México. Instituto de Ciencias del Mar y Limnología, Contribución $\mathrm{N}^{\mathrm{o}}$ 684. Universidad Nacional Autónoma de México. 17(2) 215-240.

Aragón, G.; Aguirre, M.

2014. Distribución de murciélagos en la región de Tacna (Perú). Idesia, 32(1) 119-127.

Burles, D.W.; Brigham, R.M.; Ring, A.; Reimchen. T.E. 2008. Diet of two insectivorous bats, Myotis lucifugus and Myotis keenii, in relation to arthropod abundance in a temperature Pacific Northwest rainforest environment. Canadian Journal Zoology, 86: 1.367-1.375.

Canals, M.; Grossi, B.; Iriarte-Díaz, J.; Veloso, C.

2005. Biomechanical and ecological relationships of wing morphology of eight Chilean bats. Revista Chilena de Historia Natural, 78: 215-227.

Debelica, A.; Matthews, A.K.; Ammerman, L.K.

2006. Dietary study of big free-tailed bats (Nyctinomops macrotis) in Big Bend National Park, Texas. The Southwestern Naturalist, 51(3): 414-418.

Estrada-Villegas, S.; Pérez-Torres, J.; Stevenson, P.

2010. Ensamblaje de murciélagos en un bosque subandino colombiano y análisis sobre la dieta de algunas especies. Mastozoología Neotropical, 17(1): 31-41.

Flores, M.; Calizaya, G.; Pacheco, V.; Aragón, G.

2015. Distribution of Promops davisoni Thomas, 1921 (Chiroptera: Molossidae) in Peru with a new record and southward range extension. Check List, 11(2): 1.573.

Gardner, A.L.

2008. Mammals of South America, Volume 1. Marsupials, xenarthrans, shrews, and bats. The University of Chicago Press, Chicago, 669 p.
Gillot, C.

2005. Entomología. Editorial Springer. Tercera edición. Netherlands. 783 p.

Halpern, M.; Gancz, H.; Broza, M.; Kashi, Y.

2003. Vibrio cholerae hemaglutinin/protease chironomid egg masses. Applied and Environmental Microbiology, 69(7): 4.200-4. 204 p.

Hodgkison, R.; Balding, S.T.; Zubaid, A.; Kunz, T.H.

2004. Temporal Variation in the Relative Abundance of Fruit Bats (Megachiroptera: Pteropodidae) in Relation to the Availability of Food in a Lowland Malaysian Rain Forest. Biotropica, 36(4): 522-533.

Krebs, C.J.

1999. Ecological methodology. 2nd Edition. Edit. Addison Wesley Longman. U.S.A., 745 p.

Lee, Y. \& L. Lee.

2005. Food Habits of Japanese Pipistrelles Pipistrellus abramus (Chiroptera: Vespertilionidae) in Northern Taiwan. Zoological Studies, 44(1): 95-101.

Loayza, A.P.; Ríos, R.S.; Larrea-Alcázar, D.M.

2006. Disponibilidad de recurso y dieta de murciélagos frugívoros en la Estación Biológica Tunquini, Bolivia. Ecología en Bolivia, 41(1): 7-23.

López, J.E.; Vaughan, C.

2007. Food niche overlap among neotropical frugivorous bats in Costa Rica. Revista Biológica Tropical, 55 (1): 301-313.

Lou, S.; Yurrita, C.L.

2005. Análisis de nicho alimentario en la comunidad de murciélagos frugívoros de Yaxhá, Petén, Guatemala. Acta Zoológica Mexicana, 21(1): 83-94.

Matthews, A.K.; Neiswenter, S.A.; Ammerman, L.K. 2010. Trophic ecology of the free-tailed bats Nyctinomops femorosaccus and Tadarida brasiliensis (Chriroptera: Molossidae) in Big Bend National Park, Texas. The Southwestern Naturalist, 55(3): 340-346.

Mc Gavin, G.C. 2000. Insectos, arenas y otros artrópodos terrestres. Eds. Omega, Barcelona-España, 256 p.

McWilliams, L.A.

2005. Variation in diet of the Mexican free-tailed bat (Tadarida brasiliensis mexicana). Journal of Mammalogy, 86: 599-605.

Zeballos, H.; López, E.

2002. Registro de los murciélagos de Arequipa y clave de especies. Revista de Investigación Dilloniana 2(1): 143-154. 